\title{
List of Reviewers - Brazilian Review of Finance - RBFin
}

A good quality scientific publication depends on the engagement of qualified academics. The editors of RBFin would like to thank the 53 individuals below who concluded voluntary evaluations of articles in 2013. Nine of them are associated to universities outside Brazil. Reviewers perform one review per year, with the exception of follow-up reviews of the same article. We show the number of papers reviewed in parenthesis to recognize those who gracefully exceeded this limit.

\author{
Alencar, Leonardo Soriano, Central Bank of Brazil, Brazil \\ Almeida, Vinício de Souza e, Federal University of Rio Grande do Norte, Brazil \\ Amaral-Baptista, Márcio, University Institute of Lisbon, Portugal \\ Barbedo, Claudio Henrique, Central Bank of Brazil and Ibmec Business School, Brazil \\ Barros, Lucas Ayres B. De C., University of São Paulo, Brazil \\ Bortoluzzo, Adriana Bruscato, Insper - Institute of Education and Research, Brazil \\ Bressan, Aureliano Angel, Federal University of Minas Gerais, Brazil \\ Bueno, Rodrigo de Losso da Silveira, University of São Paulo, Brazil \\ Camilo-da-Silva, Eduardo, Fluminense Federal University, Brazil \\ Carmona, Charles Ulises de Montreuil, Federal University of Pernambuco, Brazil \\ Carrasco, Vinicius Nascimento, Pontifical Catholic University of Rio de Janeiro, Brazil \\ Carvalhal-da-Silva, André Luiz, BNDES and Pontifical Catholic University of Rio de Janeiro, Brazil \\ Carvalho, José Raimundo, Federal University of Ceará, Brazil \\ Castro Jr., Francisco Henrique Figueiredo de, University of São Paulo, Brazil \\ Ceretta, Paulo Sergio, Federal University of Santa Maria, Brazil \\ Coelho, Luís Manuel Soares, Algarve University, Portugal \\ Duarte Jr., Antonio Marcos, Ibmec Business School Rio de Janeiro, Brazil \\ Eid Jr., William, Getulio Vargas Foundation São Paulo, Brazil \\ Ely, Regis Augusto, Federal University of Pelotas, Brazil \\ Esperança, José Paulo, University Institute of Lisbon, Portugal \\ Fajardo-Barbachan, José Santiago, Getulio Vargas Foundation Rio de Janeiro, Brazil \\ Frascaroli, Bruno Ferreira, Federal University of Paraíba, Brazil \\ Gaglianone, Wagner Piazza, Central Bank of Brazil, Brazil \\ Galdi, Fernando Caio, Fucape Business School, Brazil \\ González-Ferrero, Maximiliano, University of the Andes, Colombia \\ Iquiapaza-Coaguila, Robert Aldo, Federal University of Minas Gerais, Brazil \\ Klotzle, Marcelo Cabús, Pontifical Catholic University of Rio de Janeiro, Brazil \\ Lauretti, Carlos Marcelo, Presbiterian University Mackenzie, Brazil \\ Leal, Ricardo Pereira Câmara, Federal University of Rio de Janeiro, Brazil (2) \\ Matos, Paulo Rogério Faustino, Federal University of Ceará, Brazil \\ Medeiros, Otávio Ribeiro de, National University of Brasília, Brazil \\ Meurer, Roberto, Federal University of Santa Catarina, Brazil \\ Minardi, Andrea M. A. F., Insper - Institute of Education and Research, Brazil \\ Moura, Marcelo Leite, Insper - Institute of Education and Research, Brazil \\ Nabar, Sandeep, Oklahoma State University, USA \\ Nakamura, Wilson, Mackenzie University, Brazil \\ Nazaré, Ronaldo, University of Southampton, United Kingdom \\ Peixinho, Rúben M. T., Algarve University, Portugal \\ Pinho, Frank Magalhães de, Federal University of Minas Gerais, Brazil \\ Porto, Rogério, Banco do Brasil, Brazil \\ Righi, Marcelo Brutti, Federal University of Santa Maria, Brazil \\ Robles-Fernández, M. Dolores, Complutense University of Madrid, Spain \\ Rochman, Ricardo Ratner, Getulio Vargas Foundation São Paulo, Brazil \\ Rogers-Silva, Pablo, Federal University of Uberlândia, Brazil \\ Rossoni, Luciano, Grande Rio University, Brazil \\ Saito, Richard, Getulio Vargas Foundation São Paulo, Brazil \\ Sanematsu, Flávio Cysneiros, Petrobras, Brazil \\ Sant'Anna, Pedro Henrique C., Carlos III University of Madrid, Spain \\ Sobreira-Bezerra, Rogério, Getulio Vargas Foundation Rio de Janeiro, Brazil \\ Terra, Paulo Renato Soares, Federal University of Rio Grande do Sul, Brazil \\ Varga, Gyorgy, FCE Consultoria, Brazil \\ Zani, João, Unisinos, Brazil \\ Ziegelmann, Flávio Augusto, Federal University of Rio Grande do Sul, Brazil
}

Rio de Janeiro, February 28, 2014. 